\title{
Correlated nucleons in configuration space
}

\author{
Herbert Müther \\ Institut für Theoretische Physik, Universität Tübingen, Tübingen, Germany \\ Ingo Sick \\ Dept. für Physik und Astronomie, Universität Basel, Basel, Switzerland
}

(Dated: November 5, 2018)

\begin{abstract}
Several recent studies have dealt with the effects of short range correlations on the momentum distribution of nucleons in nuclei. Here we investigate the correlation effects on the density and spectral distribution in coordinate space. A combination of experimental data and spectral functions calculated from realistic N-N interactions allows us to resolve a recently uncovered discrepancy with occupation of quasi-particle states derived from (e,e'p) data.
\end{abstract}

PACS numbers: 25.30.Bf,25.30.Dh,25.30.Fj,21.60.-n

\section{INTRODUCTION}

Much of the understanding of atomic nuclei is based on the assumption that nucleons move, independently from each other, in the average potential created by the interaction with all other nucleons. A more fundamental approach to the understanding of nuclei has to start from the underlying nucleon-nucleon $(\mathrm{N}-\mathrm{N})$ interaction. Realistic models of the $\mathrm{N}-\mathrm{N}$ interaction exhibit a strongly repulsive central interaction at small inter-nucleon distances and a strong tensor component. These features lead to properties of nuclear wave functions that are beyond what is describable in terms of Independent Particle (IP) motion. In particular, strong short-range correlations (SRC) are expected to occur.

The effects of the short-range correlations are known for systems where an accurate solution of the Schrödinger equation for a realistic N-N interaction can be obtained 1]. Very light nuclei (today up to $A \leq 10$ ) and infinite nuclear matter are amongst the systems where this is feasible 2, 3, 4]. The corresponding calculations show that in a microscopic description of nuclear systems the short-range and tensor parts of the $\mathrm{N}-\mathrm{N}$ interaction have a very important, not to say dominating, influence without which not even nuclear binding can be explained.

Due to these short-range correlations the momentum distributions of nucleons acquire a tail extending to very high momenta $k$ and part of the strength, located in IP descriptions at low excitation energy $E$, is moved to very high excitation energies.

In the past, most experimental investigations were confined to rather low momenta and energies, i.e. to the region where the strength is dominated (but not entirely given) by the IP properties. In this region, the consequences of short-range correlations are indicated primarily by a depopulation of states in comparison to the predictions of IP models (including the long-range correlations which can be described by configuration mixing). According to the calculations mentioned above, a depopulation of the order of $20 \%$ is expected.

¿From the experimental information available up to now, the depopulation of IP strength at low $k, E$ is established [1] (for a caveat see below). Much less is known from a direct measurement of the strength of the spectral function $S(k, E)$ at large $k$ and $E$. A recent (e,e'p) experiment, performed at high momentum transfer $q$ in parallel kinematics by Rohe et al., provides the first direct measurement [5, 6].

This correlated strength has always been discussed in $k, E$-space where (part of) it can be separated from the IP strength. In this Rapid Communication, we take an orthogonal look at the correlated strength and discuss it in coordinate space ( $\mathrm{r}$-space). We address this question from both the theory and experiment side.

The trigger for this study lies in difficulties experienced in the past in interpreting data in terms of IP models. For example, fits with IP wavefunctions of the nuclear charge density often yielded form factors (of IP dominated transitions) with incorrect $q$-dependence. Fits with IP wave functions also have difficulties to reproduce the total densities in the nuclear interior. The origin of these difficulties: total densities have contributions from correlated nucleons that do not appear in observables dominated by quasi-particle properties. The correlated nucleons presumably have a different radial distribution.

The goal of this paper is to derive quasi-particle (QP) and correlated distributions in r-space using Green'sfunction theory. We compare the results to the correlated density in r-space which we obtain from the difference of the density - known from elastic electron scattering and the QP contributions known from (e,e'p) reactions.

As a side-product, this study also sheds light on a recently uncovered problem with QP occupation numbers derived from (e,e'p) experiments with low and high $q$, respectively [7].

\section{SINGLE-PARTICLE SPECTRAL FUNCTION}

The evaluation of the single-particle spectral functions for ${ }^{12} C$, the nucleus we use for our study, has been performed within the framework of the Green's function method [8] using the techniques described in [9, 10, 11]. 
The nucleon self-energy $\Sigma_{l j}\left(p_{m}, p_{n}, E\right)$ is determined in a discrete basis of Bessel functions $\phi_{p_{m} l j}(r)$ with appropriate boundary conditions at the surface of a spherical box with radius $R_{\text {box }}$. These basis states are identified by the angular momentum quantum numbers $l$ and $j$ and a radial quantum number $\left(p_{m}, p_{n}\right)$ referring to the momentum. For a box radius $R_{\text {box }}$ of typically $20 \mathrm{fm}$, it turns out to be sufficient to include around 60 basis states for each partial wave.

The self-energy contains a Hartree-Fock contribution $\Sigma_{l j}^{\mathrm{HF}}$ calculated in terms of a nuclear matter $G$-matrix plus complex correction terms, $\Delta \Sigma_{l j}$, which account for the inclusion of two-particle one-hole and two-hole oneparticle contributions. These correction terms are calculated directly for the finite nucleus ${ }^{12} C$, describing the intermediate particle states by plane waves orthogonalized with respect to the occupied hole states. This is a good approximation to describe the effects of SRC, however, it tends to underestimate the spectral strength due to long-range correlations at missing energies slightly above the two-hole one-particle threshold.

The single-particle Green's function is determined from this self-energy $\Sigma_{l j}^{\mathrm{HF}}+\Delta \Sigma_{l j}$ by solving the Dyson equation in the box basis described above. From the imaginary part of this Green's function one can calculate the spectral function in this basis [1] or determine it in configuration space by the transformation

$$
S_{l j}\left(r, r^{\prime} ; E\right)=\sum_{m, n} \phi_{p_{m} l j}(r) S_{l j}\left(p_{m}, p_{n} ; E\right) \phi_{p_{n} l j}^{*}\left(r^{\prime}\right),
$$

using the Bessel functions $\phi_{p_{m} l j}(r)$ described above. The spectral function can be split into the QP contribution $S_{l j}^{Q P}$, which only occurs in the $s_{1 / 2}$ and $p_{3 / 2}$ partial waves, and in the continuum contribution, $S_{l j}^{\text {cont }}$, which originates from the imaginary components in the selfenergy. This leads to the single-particle density

$$
\begin{aligned}
\rho(r) & =\sum_{l j} S_{l j}^{Q P}(r, r)+\sum_{l j} \int_{\varepsilon_{2 h 1 p}}^{\infty} d E S_{l j}^{c o n t}(r, r ; E) \\
& =\rho_{Q P}(r)+\rho_{\operatorname{corr}}(r)
\end{aligned}
$$

where the integration over missing energies $E$ starts at the threshold of two-hole one-particle configurations. We have assigned the label corr to the part of the singleparticle density, which originates from the continuum part of the spectral function to indicate that this correlated density is absent in the IP model.

Results for these contributions to the point density of protons in ${ }^{12} \mathrm{C}$ are displayed in Fig. 11 The calculation of these densities have been performed using the CD-Bonn potential for the N-N interaction [12]. A fraction of the proton density, which accounts for around 5 protons, is described by the QP part and the rest is covered by the correlated density.

In order to allow for a better comparison of the radial shape of the density contributions, Fig. 11 also contains the correlated density $\rho_{\text {corr }}(r)$ multiplied by a factor of 3 .
The comparison shows quite clearly that the correlated density is located much more in the center of the nucleus than the QP contribution.

The correlated single-particle density is distributed over partial waves $l j$ including those which are unoccupied in the IP model. A large fraction of the correlated strength, however, is contained in partial waves with $l=0$ (around 31\%) and $l=1$ (around 37\%). One also should note that, contrary to what one naively could expect, the strength in the higher $l$ states does not contribute at large $r$; the corresponding large values of $E$ pull the radial wave functions to lower $r$.

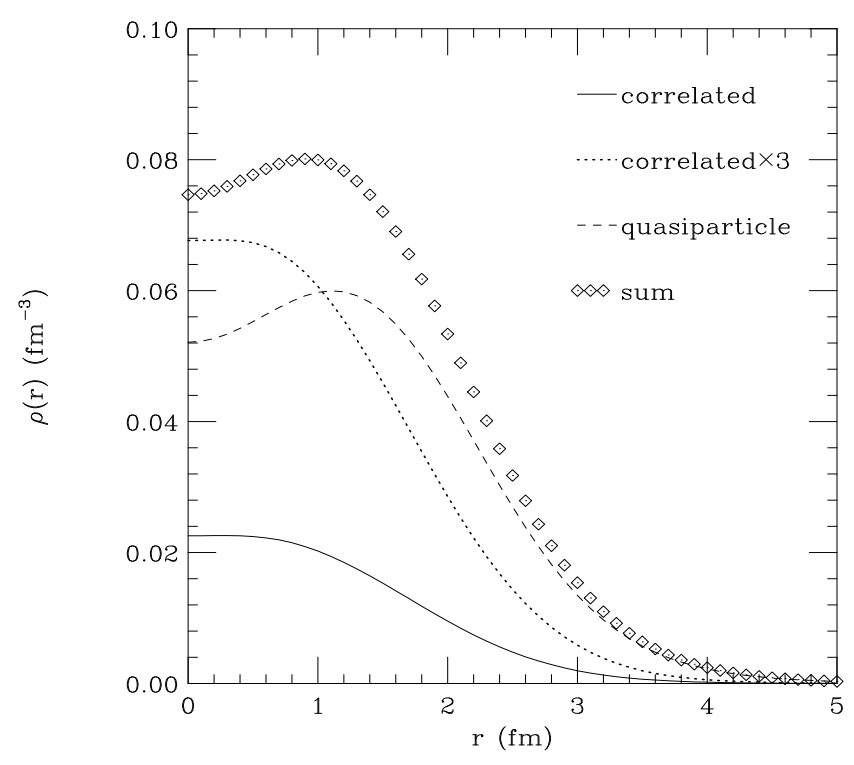

FIG. 1: Comparison of QP and correlated densities from theory.

\section{DENSITIES FROM $(e, e)$}

For ${ }^{12} \mathrm{C}$ an extensive set of elastic electron scattering data is available 13, 14, 15, 16] covering the range of momentum transfer between 0.13 and $3.7 \mathrm{fm}^{-1}$. The Carbon $r m s$-radius is precisely known from a $\mu$-X-ray experiment [17].

These data have been used to determine a modelindependent charge density using the SOG-approach of [18]. The procedure employed has been described in [19]. This yields the charge density as a function of $r$, together with an error bar that covers both the statistical and systematic uncertainties of the data, as well as the modelerror.

In order to obtain the point density, we have unfolded the effect of the finite size of the proton and neutron charge density. This has been done by parameterizing the point density, folding it with $\rho_{p}(r)+\rho_{n}(r)$ and fitting the resulting density to the charge density as determined above. The contribution of the electro-magnetic spinorbit term to the charge density turned out to be neg- 
ligible. The resulting folded density agrees within $\sim 1 \%$ with the one given in 20 .

The resulting point density is shown in figs. 203 The error bars in general are too small to be seen.

\section{QP ORBITS FROM $\left(e, e^{\prime} p\right)$}

For Carbon, quite an extensive set of (e,e'p) data is available $21,22,23,24,25,26,27,28]$; a compilation is discussed in [7]. Part of this data has been taken at low $q$ with the goal to determine the $1 \mathrm{p}$ and 1 s quasiparticle momentum distributions and occupation numbers. Some data have been taken at large $q$ mainly in connection with the determination of nuclear transparencies for high-energy protons.

The low- $q$ data, taken with good energy- and momentum resolution mainly at NIKHEF and Saclay, have been analyzed in the framework of DWBA using optical potentials known from proton-Carbon scattering. The $\mathrm{QP}$ radial wave functions have been parameterized using Woods-Saxon (WS) potentials. Lapikas et al. 7] have made a coherent analysis of the entire data set. The occupation of the QP orbits, obtained by summing the experimental spectroscopic factors, turns out to be rather low in comparison to what is known for other nuclei [1]; the summed $1 \mathrm{p}$ plus $1 \mathrm{~s}$ strength amounts to 3.4 protons only ( $56 \%$ occupation).

The high- $q$ data, determined in part with moderate energy- and momentum resolution, were taken mainly at SLAC and JLAB. The data were summed over a large region of initial momentum $k$ and removal energy $E$, and fitted using WS radial wave functions and theoretical transparencies. When using the most reliable transparencies, confirmed by other experiments, Lapikas et al. found a much higher occupancy of the QP orbits, 5.0-5.6 protons ( $\sim 87 \%$ occupation).

This discrepancy - which is very embarrassing to the practitioners of (e,e'p) as it sheds serious doubts on the quantitative interpretation of (e,e'p) data - obviously needs to be better understood, and is discussed in more detail below. This difference also has led to speculations about $q$-dependent QP-occupations, for which we see no physical basis.

A partial reason for the difference between the low$q$ and high- $q$ results is immediately clear: The low- $q$ data cover the region of missing momenta of typically $<180 \mathrm{MeV} / \mathrm{c}$ and missing energy $E<50 \mathrm{MeV}$, the high- $q$ data extend to $300 \mathrm{MeV} / \mathrm{c}$. The high- $q$ data also cover a larger range in missing energy, they are integrated up to typically $80 \mathrm{MeV}$. In this larger $k, E$-range, there is not only QP strength, but also a fraction of the correlated strength is integrated over. Before making a valid comparison, this correlated strength needs to be removed.

In order to correct for this effect, we start from the high- $q$ (e,e'p) data taken in a recent JLAB experiment [5] in quasi-elastic kinematics, which minimize final state interactions FSI and meson exchange current contribu- tions MEC. This experiment yields, in agreement with the previous JLAB and SLAC experiments, 5.2 protons in the integration region $\mathrm{E}<80 \mathrm{MeV}, \mathrm{k}<300 \mathrm{MeV} / \mathrm{c}$. This number we correct for the continuum contribution using the calculated spectral function discussed above. With this correction the discrepancy between the low- $q$ and high- $q$ results is significantly reduced; the QP occupations now are 3.4 vs 4.5 protons for the low- $q$ /high- $q$ data, respectively. Given the uncertainties of these numbers believed to perhaps $10 \%$ - there still is a worrisome incompatibility.

In order to proceed, we have to choose. We have decided to use the occupation coming from the high- $q$ measurements, as we judge the interpretation of these data to be more safe. The low- $q$ data suffer from uncertainties in the treatment of the final state interaction. Due to the low energies of the outgoing proton $(70 \mathrm{MeV}$ for the NIKHEF data 25]) coupled channel effects not treated in the usual DWBA analysis should be relevant. Van der Steenhoven et al [25, 29] have shown that inclusion of these effects would increase, for the rather soft nucleus ${ }^{12} C$, the QP occupation by $\sim 20 \%$. For the kinematics of the low- $q$ experiments, the calculations of Boffi et al. 30, 31] also predict significant MEC effects that would lead to a further increase of the QP occupation.

The value for the QP occupation deduced from the high- $q$ measurement is also compatible with the correlated strength measured directly in the recent experiment by Rohe et al. [5]. This measurement agrees with theoretical predictions for the correlated strength of $\sim 20 \%$. The summed QP strength from the low- $q$ (e,e'p) data (which thus includes the fragmentation due to long-range correlations), on the other hand, would correspond to $>40 \%$ correlated strength, i.e. be unrealistically high. Furthermore, as we will see below, our choice of the QP occupation is confirmed by a consistency check in our analysis.

Before proceeding to the calculation of the QP density, one more effect of correlations must be removed from the results of Lapikas et al. While the p-strength is located in discrete states where no ambiguity occurs, the s-strength is located in the continuum between 20 and $\sim 50 \mathrm{MeV}$ removal energy. In this region, also the correlated strength contributes, and affects the shape of the fitted WS momentum distribution. The correlated strength has a momentum distribution that falls much more slowly with increasing $k$ than the 1s QP strength; when fitting the sum with a WS parameterization, the resulting WS momentum distribution extends somewhat too far in momentum, i.e. it would have too small a radial extension in $r$-space.

We have used the theoretical 1s QP and correlated strength in the region used in [7] for the determination of the 1s momentum distribution to calculate a correction to the WS parameterization fitted to the sum. We find that the extent of the wave function in k-space needs to be reduced by $11 \%$. This modified WS-shape has been confirmed 32] by an independent analysis employing the 
recent JLAB data of [5] and the correlated spectral function of 3 .

With these QP radial wave functions, and occupations renormalized to the one derived above from the high- $q$ experiments, we can compute the QP density in $r$-space. As the radial wave functions fitted by Lapikas et al. refer to relative coordinate between the proton and the $\mathrm{CM}$ of the $(\mathrm{A}-1)$ system, we need to rescale the radial coordinate by a factor $11 / 12$, with the corresponding adjustment in height to conserve the normalization.

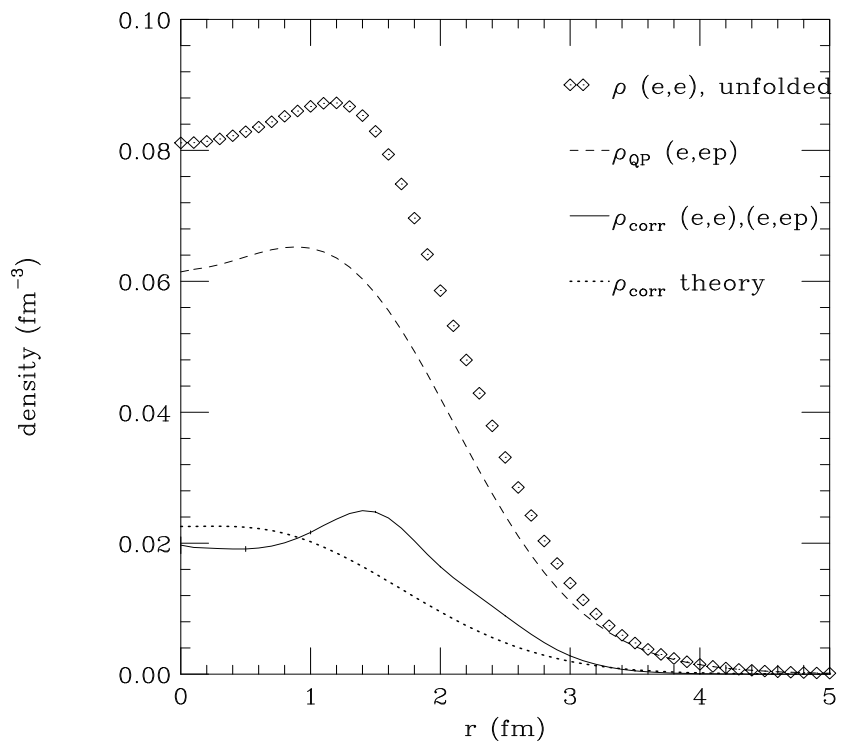

FIG. 2: Comparison of QP and correlated densities.

The resulting QP density is shown in figs. 23. The difference of the ${ }^{12} C$ point density and this QP density yields the correlated density also shown in Figs. 23] The 'error bar' on the correlated density is not straightforward to calculate due to the various adjustments that had to made in the analysis; $\pm 20 \%$ for $r<3 \mathrm{fm}$ is probably a realistic estimate.

\section{COMPARISON OF QP AND CORRELATED DENSITIES}

The first observation one can make from Figs. 23] concerns the fact that like in the theoretical analysis of Fig. 1 also the correlated density deduced from experimental data is significantly more concentrated towards the nuclear interior than the QP density. One also observes that the correlated density in the nuclear interior gives a very significant contribution, of order $30 \%$, of the central density, i.e. larger than one could have expected from the number of $20 \%$ or so of correlated nucleons. This explains why attempts to explain the total densities in terms of QP orbitals cannot be very successful.

Figure 3 shows another important feature: The experimental QP density at large $r$ agrees perfectly with

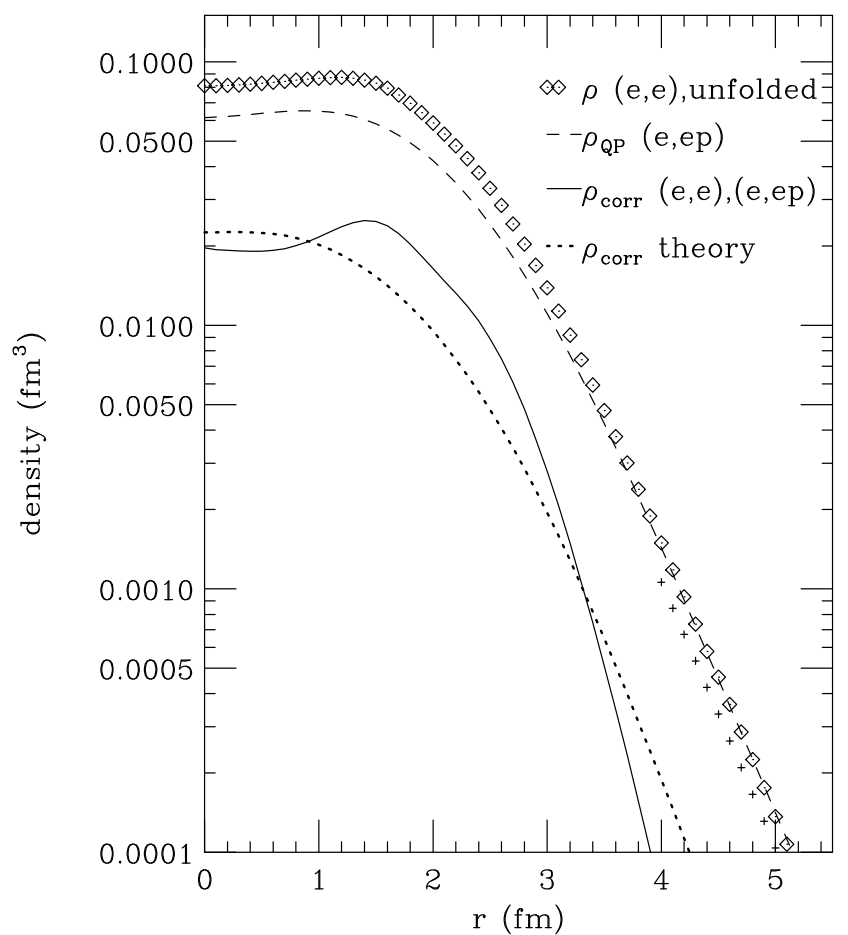

FIG. 3: Comparison of QP and correlated densities. The crosses indicate the tail of the density obtained when using the low- $q$ QP occupation.

the point density measured via elastic electron scattering. Such an agreement should occur, as at large $r$ outside the range of the nuclear potential - the density is entirely given by the tail of the least-bound QP orbit, the $1 p_{3 / 2}$ state in ${ }^{12} C$. More deeply bound states, or correlated nucleons with large removal energy, cannot contribute.

The good agreement between QP density and point density at large $r$ also confirms the correctness of our choice of QP occupations. Had we used the occupation derived from the low- $q$ experiments and, as above, the shape of $\mathrm{R}(\mathrm{r})$ determined in [7] from the fit to the world (e,e'p) data, we would have obtained the tail indicated in fig. 3 by the crosses. These are obviously significantly too low. This comparison thus provides an a posteriori justification of our procedure.

In figs. 23] we also show the correlated density obtained by theory. Considering the above-mentioned uncertainty of the experimental result, we consider the agreement between theory and experiment as a good one. The size of the correlated contribution in the nuclear interior is very similar, the rapid fall-off of the correlated density at large $r$ also agrees within our estimated uncertainty. The correlated density deduced from experimental data seems to contain more spectral strength in partial waves with $l>0$ than the theoretical one.

The large contribution of the correlated density in the nuclear interior shows that the neglect of this correlated contribution in the standard IP calculations (e.g. all the 
shell-model descriptions) is not justified.

\section{CONCLUSIONS}

Starting from (e,e'p) data we have constructed the QP density for ${ }^{12} C$ in coordinate space. The difference to the total density, obtained from elastic electron scattering, provides the density distribution of the correlated nucleons. We find that it is significantly more concentrated towards the nuclear interior. We also find good agreement with the theoretical calculation of the correlated density distribution.

The large contribution of the density related to shortrange $\mathrm{N}-\mathrm{N}$ correlations, $\sim 30 \%$ in the nuclear interior, together with the fact that the shape of the correlated density differs strongly from the QP density, explains the poor performance of $\mathrm{QP}$ wave functions in explaining many observables. Due to the shape difference, the shortcoming of the neglect of the correlated contribution also cannot satisfactorily be 'compensated' by using effective quantities like effective charges, etc.

As a side product, our analysis provides a solution to the puzzle raised in [7], the pronounced disagreement between QP occupations derived from the low- $q$ and high- $q$ (e,e'p) experiments. We find that only the high- $q$ occupation (suitably corrected for the correlated contribution not considered in (7]) is compatible with the independent information from elastic electron scattering.

\section{Acknowledgments}

We would like to thank Louk Lapikas for providing the QP wave functions, Kai Hencken and Dirk Trautmann for elucidation of CM problems and Daniela Rohe for an independent determination of the 1s QP momentum distribution. This work has been supported by the DFG (Graduiertenkolleg Basel-Tübingen) and the Schweizerische Nationalfonds.
[1] V.R. Pandharipande, I. Sick, and P. deWitt Huberts. Rev. Mod. Phys., 69:981, 1997.

[2] S.C. Pieper and R.B. Wiringa. Ann. Rev. Nucl. Part. Sci., 51:53, 2001.

[3] O. Benhar, A. Fabrocini, and S. Fantoni. Nucl. Phys. A, 505:267, 1989.

[4] H. Müther and A. Polls. Prog. Part. Nucl. Phys., 45:243, 2000.

[5] D. Rohe. Habilitationsschrift, Universität Basel, 2004.

[6] D. Rohe et al. subm. to Phys. Rev. Lett., 2004.

[7] L. Lapikas, G. van der Steenhoven, L. Frankfurt, M. Strikman, and M. Zhalov. Phys. Rev. C, 61:064325, 2000.

[8] W.H. Dickhoff and C. Barbieri. Prog. Part. and Nucl. Phys., 52:377, 2004.

[9] H. Müther, A. Polls, and W.H. Dickhoff. Phys. Rev. C, 51:3040, 1995.

[10] A. Polls, H. Müther, and W.H. Dickhoff. Nucl. Phys. A, 594:117, 1995.

[11] A. Polls, M. Radici, S. Boffi, W.H. Dickhoff, and H. Müther. Phys. Rev. C, 55:810, 1997.

[12] R. Machleidt, F. Samarucca, and Y. Song. Phys. Rev., 53:R1483, 1996.

[13] I. Sick and J.S. McCarthy. Nucl. Phys. A, 150:631, 1970.

[14] L.S. Cardman, J.W. Lightbody, S. Penner, W.P. Trower, and S.F. Williamson. Phys. Lett. B, 91:203, 1980.
[15] G. Fey. Thesis, TH Darmstadt, 1973.

[16] J.A. Jansen, R.T. Peerdeman, and C. deVries. Nucl. Phys. A, 188:337, 1972.

[17] W. Ruckstuhl et al. Nucl. Phys. A, 430:685, 1984.

[18] I. Sick. Nucl. Phys. A, 218:509, 1974.

[19] I. Sick. Phys. Lett. B, 116:212, 1982.

[20] H. DeVries et al. Atomic Data, 36:495, 1987.

[21] U. Amaldi et al. Phys. Lett. B, 25:24, 1967.

[22] K. Nakamura et al. Nucl. Phys. A, 268:381, 1876.

[23] J. Mougey, M. Bernheim, A. Bussière, A. Gillebert, Phan Xuan Ho, M. Priou, D. Royer, I. Sick, and G.J. Wagner. Nucl. Phys. A, 262:461, 1976.

[24] M. Bernheim et al. Nucl. Phys. A, 375:381, 1982.

[25] G. van der Steenhoven et al. Nucl. Phys. A, 484:445, 1988.

[26] K. I. Blomqvist et al. Z. Phys. A, 351:353, 1995.

[27] N.C.R. Makins et al. Phys. Rev. Lett., 72:1986, 1994.

[28] D. Dutta et al. Phys. Rev. C, 68:064603, 2003.

[29] G. van der Steenhoven. PhD thesis, VU Amsterdam, 1987.

[30] S. Boffi and M. Radici. Nucl. Phys. A, 526:602, 1991.

[31] S. Boffi, C. Giusti, and F.D. Pacati. Phys.Rep, 226:1, 1993.

[32] D. Rohe. priv. com., 2004. 